TM-1377

0121.585

\title{
Design Charts for Spacing of Vacuum Line Supports*
}

J.L. Western and K.J. Krempetz

Fermi National Accelerator Laboratory

P.O. Box 500, Batavia, Illinois 60510

May 4, 1987 


\title{
DESION CHARTS FOR SPACING OF VACUUM LINE SUPPORTS
}

\author{
by: Jeffrey L Western and Kurt J. Krempetz
}

\section{Introduction}

This paper presents design tables and graphs to aid engineers and designers in the selection of support specing for vacuum beem lines end other vecuum pipes. This date applies to support spacing for thin wall pipes and tubes under a internal vacuum (or open to atmosphere) and subject to external atmospheric pressure. Data is generated from the equations for a simply supported thin walled pipe or tube of circular cross section.

These tables and graphs indicate what the minimum spacing of supports is for a pipe or tube of a given radius and supply date for carbon steel, stainless steel, aluminum and copper materials. The wall thickness of the pipe and tube is limited to $1 / 10$ of the pipe radius $(T \ll R / 10$ ). A thickness of $R / 10$ results in a decrease in the length values in the attached tables of 108, whereas a thickness of $R / 100$ results in 8 decrease in the length values in the attached tables of 18.

Snow loods, wind loads, equipment loeds, etc. are not included in the tables and graphs. This dato is applicable for vacuum pipes or tubes under its own static dead weight.

\section{Griteria Used For Tables And Graphs}

The besic loedings to be considered in the selection of support spacing for vacuum system pipe or tube runs is the weight of the pipe or tube (not including the weight of the fittings and valves) and what deflection or stress is acceptable. The moments and reactions caused by these loads can be computed by the law of statics. The spacing of supports is governed by: 1) The acceptable deflection of the pipe and 2) The allowable siress in the pipe.

The worst case is a simply supported beam uniformly loaded. The static equations that govern the problem are: 2

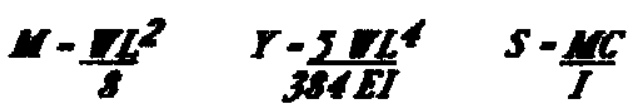

$$
\text { Where } \quad \begin{aligned}
\boldsymbol{M} & =\text { Bending Moment } \\
\boldsymbol{L} & =\text { Length of Pipe } \\
\boldsymbol{I} & =\text { Uniform Loed in LBS/IN } \\
\boldsymbol{E} & =\text { Modulus of Elasticity } \\
\boldsymbol{I} & =\text { Moment of Inertio } \\
\boldsymbol{S} & =\text { Bending Stress } \\
\boldsymbol{C} & =\boldsymbol{R} \text { Where } \boldsymbol{R}=\text { Redius of Pipe }
\end{aligned}
$$


It can also be shown for a thin walled pipe: 3

$$
A-2 a R T \quad I=a^{3} T
$$

Where $\quad A=$ Area of Pipe

$\boldsymbol{R}=$ Redius of Pipe

$T=$ Wall Thickness of Pipe

$I=$ Moment of Inertia

$\Delta=3.141593$

Then

$$
\sigma-\frac{V P}{L}-\frac{2 a R T L P}{L}=2 a R T P
$$

Where $\quad \boldsymbol{p}=$ Density of Pipe

$\boldsymbol{F}=$ Volume of Pipe Material

Combining the Above Equations for Deflection:

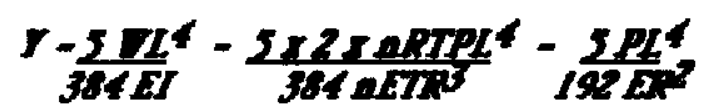

Reerranging and Solving for Length:

$$
L-\frac{\left(192 \pi x^{2}\right)^{1 / 4}}{5 P} \quad \text { (Equation 1) }
$$

Combining the Above Equations for Stress:

$$
s-\frac{T G L^{2}}{8 I}-\frac{2 a R T P L^{2}}{8 A T R^{2}}-\frac{P L^{2}}{1 R}
$$

Rearranging and Solving for Length:

$$
\left.L-\left(\frac{A S P}{P}\right) / 2 \quad \text { (Equation } 2\right)
$$

Using a computer program the attached Tables and Oraphs were generated for the following materials:
A. Carbon / Stainless Steel (Table 1 and Greph 1 )
$\boldsymbol{S}=18,750 \mathrm{PSI} ; \boldsymbol{E}=30,000,000 \mathrm{PSI} ; \boldsymbol{P}=.284 \mathrm{PCl}$
B. Copper ( Toble 2 and Graph 2 )
$\boldsymbol{S}=6,700 \mathrm{PSI} ; \boldsymbol{E}=17,000,000 \mathrm{PSI} ; \boldsymbol{P}=.322 \mathrm{PCl}$
C. Aluminum ( Table 3 and Graph 3 )
$\boldsymbol{S}=10,000 \mathrm{PSI} ; \boldsymbol{E}=10,000,000 \mathrm{PSI} ; \boldsymbol{P}=.100 \mathrm{PCl}$




\section{Precautions}

The following precautions should be observed:

1. Tables and Grephs only apply for material that hes a minimum physical property better than those shown above.

2. The pipe is carrying no internal fluid.

3. The pipe has no external loed other than its own static dead weight and is not subject to any dynamic loed.

4. The pipe is not subject to any wind, ice or snow loads.

5. No provisions were included for fittings or valves that may cause higher loadings on the pipe.

6. These Tables and Graphs are for pipes and tubes that are under vacuum (or open to atmosphere) and subject to external atmospher ic pressure.

7. Thickness is limited to $1 / 10$ of the redius $(T / R / 10)$.

\section{Intented Use of Tohles And Graphs}

These tables and graphs are intended to give deflection and stress information for simply supported lengths of round pipe and tubes under self weight static condition only.

This data is not intended for pipes and tubes under maximum stress or deflection condition. Extremely long pipes and tubes should be enalyzed in more detail.

\section{Bibliooreptiy}

1American Institute of Steel Construction. Menual of Steel

Construction, Eighth Edition. p. 2-114

2 Amer ican Institute of Steel Construction, Menual of Steel

Construction, Eighth Edition. p. 2-114

3 Roark, Raymond J. and Young, Warren C., Formules for Stress and

Strain, Fifth Edition, p. 66

\section{Acknowlechements}

We wish to acknowledge the efforts made by Julie Heim and John Rauch in the preparation of these tables and graphs. 
ILAPIF NO I

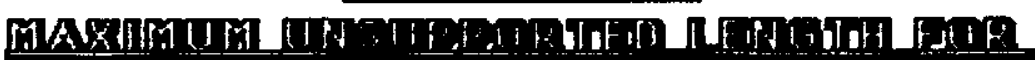

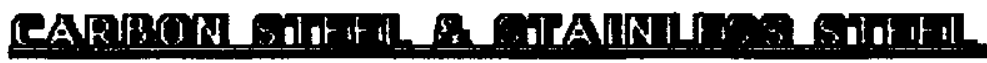

\begin{tabular}{|c|c|c|c|c|c|c|c|c|c|c|}
\hline \multirow{2}{*}{$\begin{array}{c}\text { PIPE } \\
\text { OR } \\
\text { TUBE } \\
\text { RADIUS } \\
\text { (INCHES) } \\
\end{array}$} & \multicolumn{10}{|c|}{$\begin{array}{l}\text { MAXIMUM UNSUPPORTED LENGTH OF PIPE (FEET) FOR } \\
\text { ACCEPTABLE DEFLECTION }(\triangle) \text { OR ALLOWABLE STRESS (O) }\end{array}$} \\
\hline & $\Delta=1 / 16$ & $\Delta=1 / 8$ & $\Delta=1 / 4$ & $\Delta_{=1 / 2}$ & $\Delta$. & $\stackrel{\Delta}{=2}$ & $\Delta=$ & $\Delta=10$ & $\Delta=$ & $\begin{array}{l}\sigma= \\
18750 \mathrm{psi}\end{array}$ \\
\hline $1 / 2$ & 7 & 9 & 11 & 13 & 15 & 18 & 22 & 26 & 29 & 30 \\
\hline 1 & 11 & 13 & 15 & 18 & 21 & 25 & 31 & 37 & 41 & 43 \\
\hline 2 & 15 & 18 & 21 & 25 & 30 & 35 & 44 & 53 & 59 & 61 \\
\hline 3 & 18 & 22 & 26 & 31 & 36 & 43 & 54 & 65 & 72 & 74 \\
\hline 4 & 21 & 25 & 30 & 35 & 42 & 50 & 63 & 75 & 83 & 86 \\
\hline 5 & 24 & 28 & 33 & 40 & 47 & 56 & 70 & 84 & 92 & 96 \\
\hline 6 & 26 & 31 & 36 & 43 & 52 & 61 & 77 & 92 & 101 & 105 \\
\hline 7 & 28 & 33 & 39 & 47 & 56 & 66 & 83 & 99 & 110 & 113 \\
\hline 8 & 30 & 35 & 42 & 50 & 60 & 71 & 89 & 106 & 117 & 121 \\
\hline 9 & 32 & 38 & 45 & 53 & 63 & 75 & 94 & 112 & 124 & 129 \\
\hline 10 & 33 & 40 & 47 & 56 & 67 & 79 & 99 & 118 & 130 & 136 \\
\hline 11 & 35 & 41 & 49 & 59 & 70 & 83 & 104 & 124 & 137 & 142 \\
\hline 12 & 36 & 43 & 52 & 61 & 73 & 87 & 109 & 130 & 143 & 148 \\
\hline
\end{tabular}


TABLENOL2

MAXIMUM UNSUPPORIIED LENGIIH,

EOR REOPPFIR

\begin{tabular}{|c|c|c|c|c|c|c|c|}
\hline \multirow{2}{*}{$\begin{array}{c}\text { PIPE } \\
\text { OR } \\
\text { TUBE } \\
\text { RADIUS } \\
\text { (INCHES) }\end{array}$} & \multicolumn{7}{|c|}{$\begin{array}{l}\text { MAXIMUM UNSUPPORTED LENGTH OF PIPE (FEET) FOR } \\
\text { ACCEPTABLE DEFLECTION }(\Delta) \text { OR ALLOWABLE STRESS }(\sigma)\end{array}$} \\
\hline & $\Delta_{1 / 16}$ & $\Delta=1 / 8$ & $\Delta_{1 / 4}$ & $\Delta_{=1 / 2}$ & $\Delta$. & $\Delta=2$ & $\begin{array}{l}\sigma= \\
6700 \text { psi }\end{array}$ \\
\hline $1 / 2$ & 6 & 7 & 9 & 11 & 13 & 15 & 17 \\
\hline 1 & 9 & 11 & 13 & 15 & 18 & 21 & 24 \\
\hline 2 & 13 & 15 & 18 & 21 & 25 & 30 & 34 \\
\hline 3 & 15 & 18 & 22 & 26 & 31 & 36 & 42 \\
\hline 4 & 18 & 21 & 25 & 30 & 35 & 42 & 48 \\
\hline 5 & 20 & 24 & 28 & 33 & 40 & 47 & 54 \\
\hline 6 & 22 & 26 & 31 & 36 & 43 & 52 & 59 \\
\hline 7 & 23 & 28 & 33 & 39 & 47 & 56 & 64 \\
\hline 8 & 25 & 30 & 35 & 42 & 50 & 59 & 68 \\
\hline 9 & 27 & 32 & 38 & 45 & 53 & 63 & 72 \\
\hline 10 & 28 & 33 & 40 & 47 & 56 & 67 & 76 \\
\hline 11 & 29 & 35 & 41 & 49 & 59 & 70 & 80 \\
\hline 12 & 31 & 36 & 43 & 52 & 61 & 73 & 83 \\
\hline
\end{tabular}


IAABUR NO 3

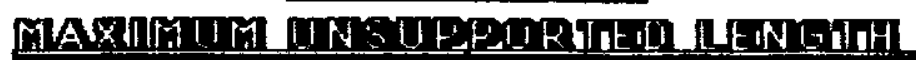

ITR AUIMINIIM

\begin{tabular}{|c|c|c|c|c|c|c|c|c|c|c|}
\hline \multirow{2}{*}{$\begin{array}{c}\text { PIPE } \\
\text { OR } \\
\text { TUBE } \\
\text { RUDIUS } \\
\text { (INCHES) }\end{array}$} & \multicolumn{10}{|c|}{$\begin{array}{l}\text { MAXIMUN UNSUPPORTEDED LENGTH OF PIPE (FEET) FOR } \\
\begin{array}{llll}\text { ACCEPTABLE DEFLECTION } & (\Delta) \text { OR ALLOWABLE STRESS }(\sigma)\end{array}\end{array}$} \\
\hline & $\Delta=1 / 16$ & $\Delta_{1 / 8}$ & $=1 / 4$ & $\Delta_{1 / 2 *}$ & $\widehat{\Delta}$ & $\Delta$ & $\Delta$ & $=10$ & $\Delta=15$ & $\begin{array}{l}\sigma^{\sigma}= \\
10000 \text { psi }\end{array}$ \\
\hline $1 / 2$ & 7 & 9 & 10 & 12 & 15 & 17 & 22 & 26 & 29 & 37 \\
\hline I & 10 & 12 & 15 & 17 & 21 & 25 & 31 & 37 & 41 & 53 \\
\hline 2 & 15 & 17 & 21 & 25 & 29 & 35 & 44 & 52 & 58 & 75 \\
\hline 3 & 18 & 21 & 25 & 30 & 36 & 43 & 54 & 64 & 71 & 91 \\
\hline 4 & 21 & 25 & 29 & 35 & 41 & 49 & 62 & 74 & 82 & 105 \\
\hline 5 & 23 & 28 & 33 & 39 & 46 & 55 & 69 & 82 & 91 & 118 \\
\hline 6 & 25 & 30 & 36 & 43 & 51 & 60 & 76 & 90 & 100 & 129 \\
\hline 7 & 27 & 33 & 39 & 46 & 55 & 65 & 82 & 98 & 108 & 139 \\
\hline 8 & 29 & 35 & 41 & 49 & 59 & 70 & 88 & 104 & 115 & 149 \\
\hline 9 & 31 & 37 & 44 & 52 & 62 & 74 & 93 & 111 & 122 & 158 \\
\hline 10 & 33 & 39 & 46 & 55 & 66 & 78 & 98 & 117 & 129 & 167 \\
\hline 11 & 34 & 41 & 49 & 58 & 69 & 82 & 103 & 122 & 135 & 175 \\
\hline 12 & 36 & 43 & 51 & 60 & 72 & 85 & 107 & 128 & 141 & 183 \\
\hline
\end{tabular}




\section{$\frac{\text { GRAPH NO. } 1}{\text { CARBON/STA I NLESS STEEL }}$ UNSUPPORTED LENGTH OF \\ VACUUM PIPES}

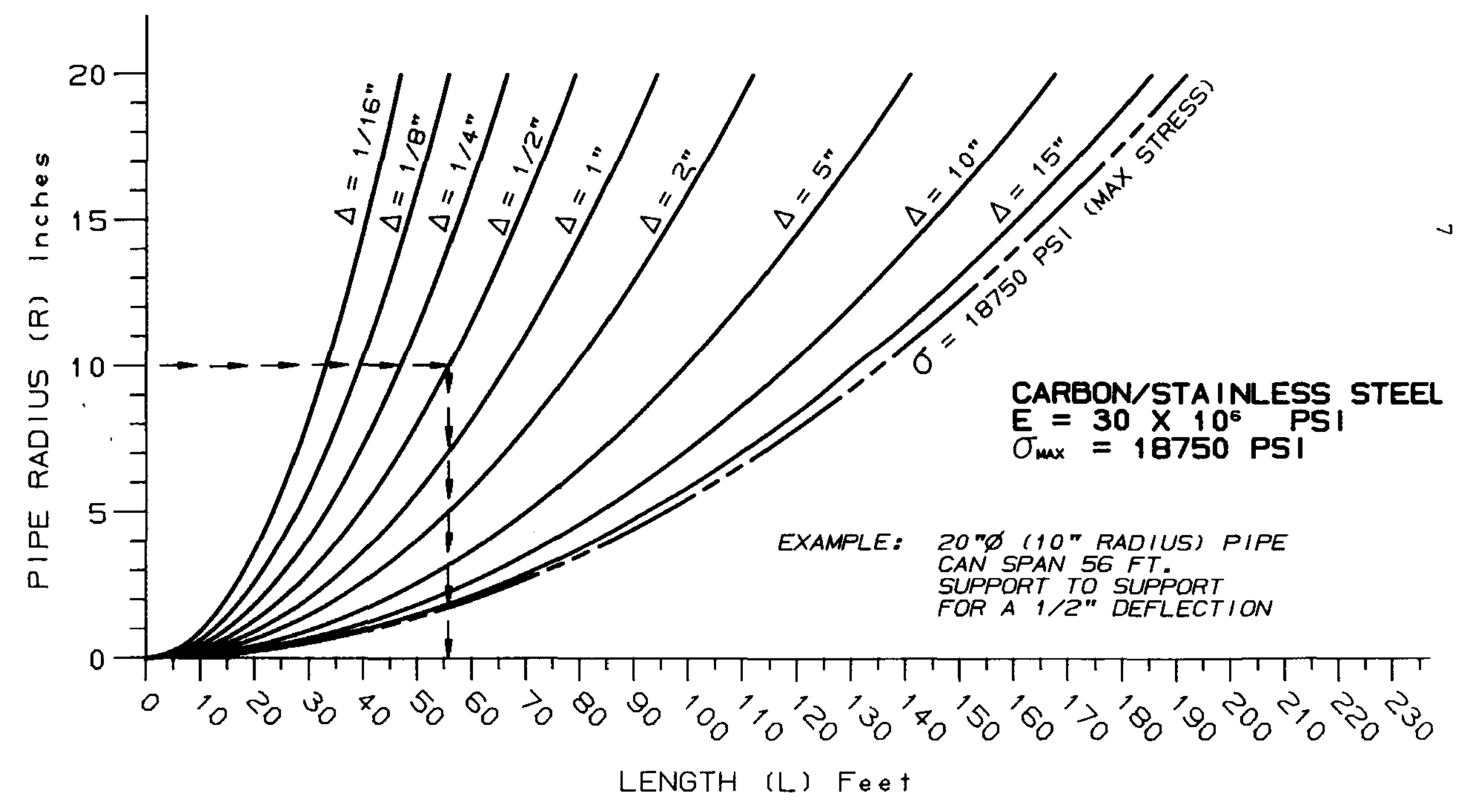




\section{$\frac{\text { GRAPH NO. } 2}{\text { COPPER }}$ \\ UNSUPPORTED LENGTH \\ $\mathrm{OF}$ \\ VACUUM PIPES}

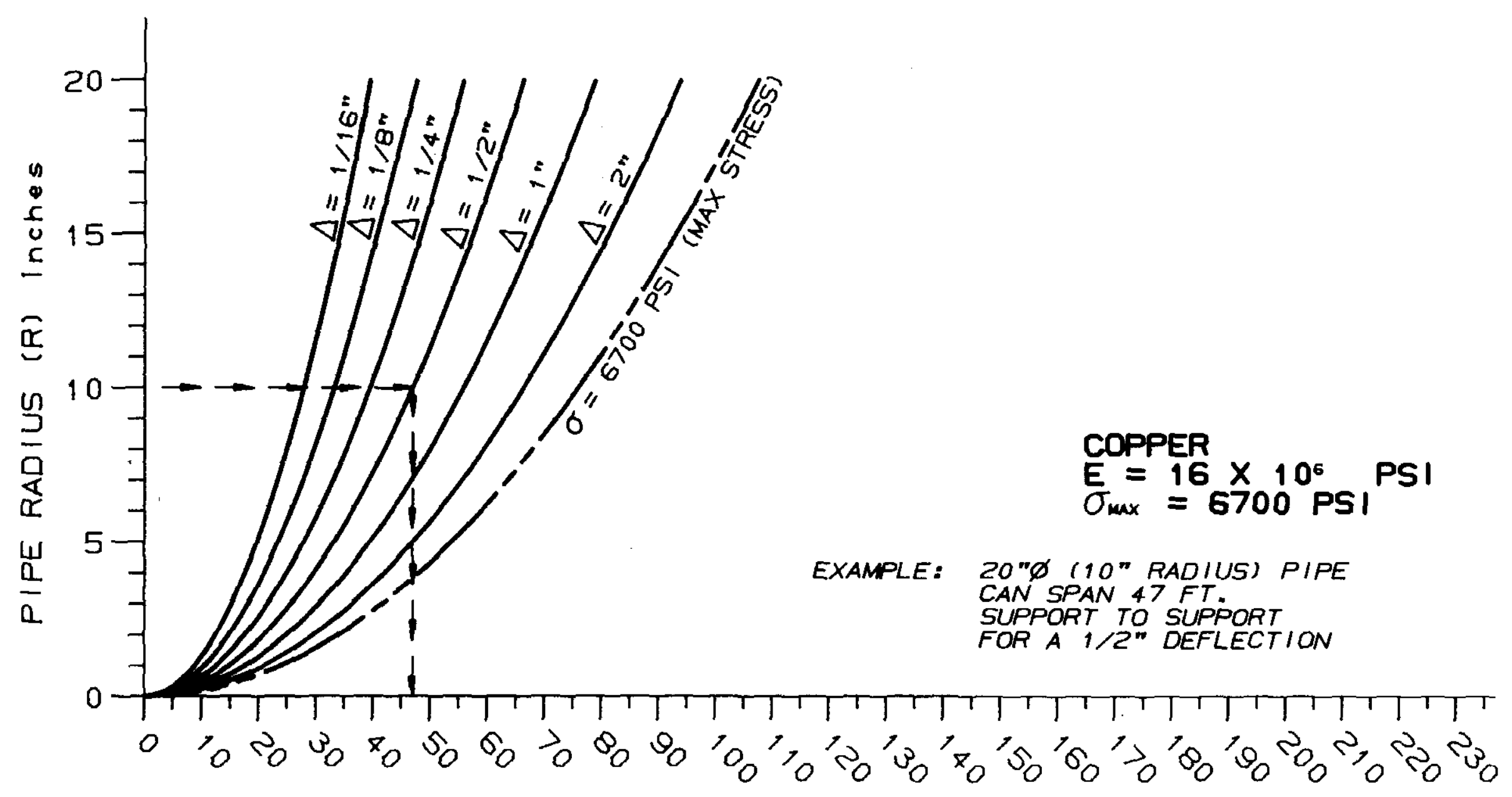

LENGTH (L) $\mathrm{Feet}$ 


\section{GRAPH NO. 3 \\ ALUM I NUM \\ UNSUPPORTED LENGTH \\ OF \\ VACUUM PIPES}

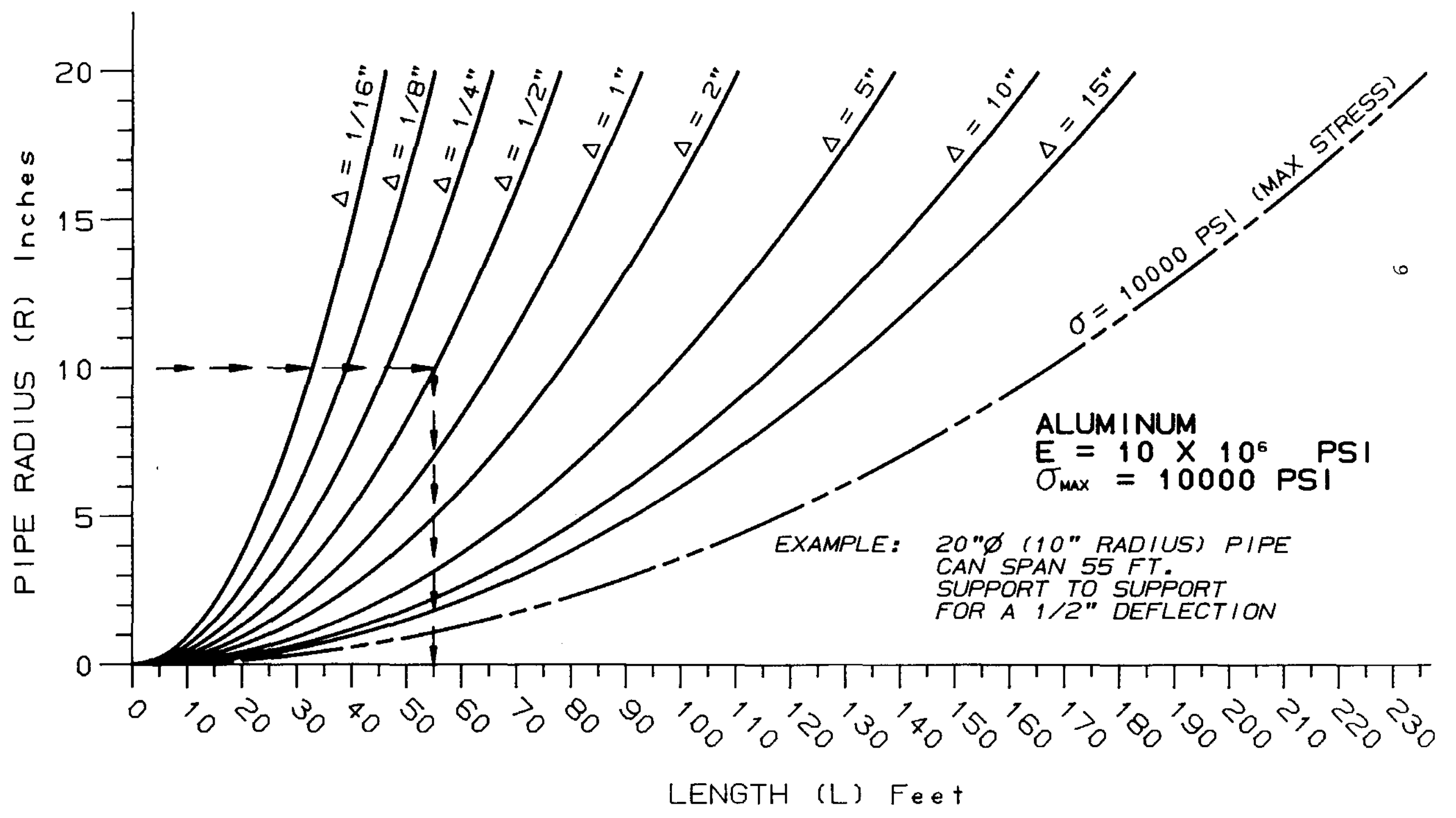

